\title{
Hinweise zu den Vordrucken
}

Diese Muster wurden mit Sorgfalt und nach bestem Gewissen erstellt.

Sie stellen jedoch nur eine Arbeitshilfe und Anregung für typische Fallgestaltungen dar.

Die Eigenverantwortung für die Formulierungen trägt der Nutzer.

Der Autor übernimmt keinerlei Haftung für die Richtigkeit und Vollständigkeit der hier vorliegenden Formulierungsbeispiele und der erläuternden Ausführungen.

Bitte beachten Sie, dass Sie in jedem Fall die vorgesehenen Eintragungen vollständig vornehmen.

Die Muster sind nur für Vertragsgestaltungen in Deutschland nutzbar.

Bitte beachten Sie die kursiv gedruckten Erläuterungen. Die Fußnoten sind nicht Teil des jeweiligen Musters, sondern dienen nur der Erläuterung.

Teils finden Sie mehrere Varianten zur Auswahl. Kreuzen Sie Ihren Favoriten an oder besser - löschen Sie die nicht verwendenden Texte. Wählen Sie auf jeden Fall eine Alternative aus.

Im Zweifelsfall nehmen Sie bitte unbedingt vor Verwendung des Musters Kontakt mit uns auf. 